\title{
Appendix 1 \\ Core Events in Volumes I-III and the Contents of the Atlas Folio
}

\section{Volume I}

\section{Introduction}

Anatomical notes on the kidney

Cadaveric changes in the kidney

Pathological changes in the kidney

Classification of renal disease

How to examine the diseased kidney

Examination of ureter and bladder

Relationship of diseased kidney to other systems

Causes of renal disease

\section{The Urine}

Examination of the urine

Modification of natural constituents of the urine

Substances found in the urine incidentally

Clouds, sediments and oils in the urine

Putrefaction of the urine

History of urinary abnormalities

\section{Renal Diseases}

Trauma to the kidney

Inflammation of the kidney

Nephritis

Simple nephritis

Simple nephritis in abnormal kidneys

Simple nephritis related to age

Influence of toxic substance on the reduction of simple nephritis

Relationship of simple nephritis to other systemic diseases (See contents of Volume II under albuminous nephritis)

Plates I-VI: Microscopy of urinary sediment 


\section{Volume II}

Nephritis following poisoning

Nephritis in gangrenous infections

Nephritis in smallpox, scarlet fever and "la Rougeole"

Nephritis in yellow fever

Nephritis in typhoid fever

Nephritis in septicaemia

Gouty nephritis

Nephritis in rheumatic conditions

Albuminous nephritis - acute and chronic

Relationship of albuminous nephritis to other diseases

Relationship of albuminous nephritis to diseases of the cardiovascular system

Relationship of albuminous nephritis to diseases of the respiratory system

Relationship of albuminous nephritis to diseases of the gastro-intestinal tract

Relationship of albuminous nephritis to diseases of the central nervous system

Relationship of albuminous nephritis to diseases of the reproductive system

Relationship of albuminous nephritis to diseases of the skin and connective tissue

Relationship of albuminous nephritis to eruptive fevers

Relationship of albuminous nephritis to rheumatic diseases

Relationship of albuminous nephritis to gout

History of albuminous nephritis

Relationship of certain dropsies to disease of the kidney

Conclusions

Table 1: Coagulable urine in infants

Table 2: Appearance and specific gravity of serum in several illnesses

\section{Volume III}

Pyelitis

Relationship of pyelitis to other diseases of the kidney

Relationship of pyelitis to ureteric lesions

Relationship of pyelitis to urethral and prostatic disease

Relationship of pyelitis to bladder disease

Relationship of pyelitis to infections of the reproductive system

Relationship of pyelitis to diseases of the gastro-intestinal system

Relationship of pyelitis to infections of the nervous system

Relationship of pyelitis to infections of the spinal cord

Relationship of pyelitis to diseases of the large vessels

Relationship of pyelitis to diseases of the respiratory system

Relationship of pyelitis to fevers, gout, dropsy and diseases of the skin

History of pyelitis

Pyelonephritis

Perinephric abnormalities

Renal fistula: lumbo-renal, gastro-renal, intestino-renal, pulmono-renal 
Renal haemorrhage and haematuria

Intra-renal haemorrhage

History of renal haemorrhage and haematuria

Hyperaemia of the kidneys

Anaemia of the kidneys

Hypertrophy and atrophy of the kidneys

Hydronephrosis

Renal cysts

Diseases of renal vessels

Diseases of renal nerves

Abnormal tissue deposits in the kidney

Tuberculosis of the kidney

Cancer of the kidney

Melanotic kidneys

Colloidal deposits in the kidneys

Yellow deposits in the kidneys

Clear granulations of the kidneys

Worms and other organisms in the kidneys

Foreign bodies in the kidneys

Gangrene of the kidneys

Supernumerary kidneys

Absence of the kidneys

Abnormalities of the kidneys-position, mobility, shape and fusion

\section{Contents of the Atlas 12 Parts each with 5 Plates}

\section{Part I}

Simple Nephritis

Rheumatic Nephritis

Septic Nephritis

Part II

Albuminous Nephritis

(Maladie de Bright)

Part III

Pyelitis

Part IV

Pyelonephritis

Perinephric Abscess

Renal Fistula
Part V

Hydronephrosis

Urinary cysts

\section{Part VI}

Serous Cysts

Acephalic Cysts

Worms

Part VII

Anaemia

Hyperaemia

Atrophy

Hypertrophy 
Part VIII

Abnormalities of Shape and Position of the kidneys

\section{Part IX}

Tuberculoid Kidneys

Melanosis, etc.

Part X

Cancer of the kidneys

\section{Part XI}

Diseases of the Membranes

Vessels and Lymphatics of the Kidney

And its Collecting System

\section{Part XII}

Diseases of the Suprarenal

Capsule 